unbedingt erforderlich, da $B$ der Praktikant die jedesmal zu behandelnden Kapitel durch häusliches Studium vorbereitet; nur so kann das obengenannte Ziel erreicht werden.

F. Arndt.

Aus dem Vorwort zur zweiten Auflage.

In der vorliegenden zweiten Auflage sind etliche Versuchsvorschriften und Angaben gemäB den inzwischen gesammelten Erfahrungen verbessert, sowie die am Anfang stehenden Anweisungen für den Gebrauch der Apparate und Reagenzien wesentlich erweitert und geordnet worden.

Breslau, Januar 1919.

F. A.

\title{
Aus dem Vorwort zur dritten Auflage.
}

Von einer eingehenderen Berücksichtigung des Analysenganges wurde auch diesmal abgesehen. Wo eine solche möglich ist, kann einer der zahlreichen kurzen analytischen Leitfäden hinzugezogen werden, während es gerade für die ganz primitiven Analysen, auf die man sich aus Mangel an Zeit, Einrichtungen und Lehrkräften meist doch beschränken $\mathrm{mu} B$, an sonstigen Unterlagen fehlt. Dagegen wurden an einigen Stellen ,analytische Übungen" eingeschoben, die ohne besondere InstitutsmaBnahmen von den fotteren Praktikanten ausgeführt werden können und zur Befestigung des jeweils Gelernten sowie zur Förderung des praktischen Verständnisses nützlich sein dürften.

Breslau, Oktober 1919.

F. A.

\section{Aus dem Vorwort zur fünften und sechsten Auflage.}

Das auch hier unverändert abgedruckte Vorwort zur ersten Auflage ist kürzlich von einem Referenten dahin miBverstanden worden, da $B$ „Hinweise auf die medizinische Bedeutung der Stoffe und Reaktionen absichtlich vermieden worden" seien. Dies ist keineswegs der Fall, weder in der vorliegenden noch in den früheren Auflagen. Gemeint ist, daß bei der Auswahl des ExperimentierStoffes nur chemische und nicht medizinische Gesichtspunkte maB- 